\title{
Therapy mental and psychological troubles (MPSYT) by repeating phrases of positive thoughts (TRPPT) with women. Intercultural comparison: Middle East and Europe, war and peace. A controlled and randomized study-Cognitive therapy \& positive psychotherapy.
}

\author{
Muaweah Ahmad Alsaleh \\ Center for Research on Risks and Vulnerabilities at the University of Caen Normandy \& CHU de Caen, France. \\ University of Aleppo, Syria
}

\begin{abstract}
Background: Positive education can be one of the tools that enable the women in different aspects of her life, which produces strong and healthy women, healthy family, and so healthy society. Mental and psychological troubles are common symptoms among women. The prevalence of mental and psychological troubles among women subjected to mental and psychological troubles is the highest in psycho-difficulties. The treatment of these mental and psychological troubles is recommended among women.
\end{abstract}

Aim: The objective of this study was the first to evaluate the short-term effects of the method of Therapy by Repeating Phrases of Positive Thoughts in the war and peace.

Method: The Syrian sample consisted of 20 women from the University of Damascus, aged 19 to 27 years, and 4 women with Multiple Sclerosis from Damascus Hospital and Ibn Alnafees Hospital, aged 20 to 29 years. The French sample consisted of 33 women from the University of Caen Normandy, aged 18 to 23 years.

Results: Experimental group who benefited from treatment have lower level of depression, anxiety and stress and there were significant differences between experimental $(p<0.05)$ and control groups $(p>0.05)$. For Syrian samples, according to value of effect size (Cohen's $d>0.80)$ and standardized response mean $>\mathbf{0 . 8 0}$, negative automatic thought, depression, anxiety, stress decrease after Therapy by Repeating Phrases of Positive Thoughts; and positive automatic thought, self-esteem and satisfaction in life improve among multiple sclerosis women patients and students after Therapy by Repeating Phrases of Positive Thoughts. For French sample, according to value of effect size (Cohen's d which vary between 0.11-0.41) and standardized response mean which vary between $0.33-0.82$, depression, anxiety, stress decrease after Therapy by Repeating Phrases of Positive Thoughts. The results showed a significant improvement in psychological disturbances throughout the experimental group and a significant reduction in stress following the Therapy by Repeating Phrases of Positive Thoughts protocol, less anxiety and also less depression. The short-term effect Therapy by Repeating Phrases of Positive Thoughts is significant. Treatment and remission of mental and psychological troubles are implemented by Therapy by Repeating Phrases of Positive Thoughts. The Therapy by Repeating Phrases of Positive Thoughts improves the mental health of women.

Conclusion: The RPPT treat of depression, anxiety and stress among women subjected to MPSYT and war and improve their psychological well-being and quality of life. The Therapy by Repeating Phrases of Positive Thoughts are proposed here as a fast treatment against mental and psychological troubles. The results show improved stress control capabilities, improved mood and anxiety. Therapy by Repeating Phrases of Positive Thoughts has proved beneficial for women subjected to war and mental and psychological troubles. More subjects repeat phrases with a very positive sense, more depression, anxiety and stress decrease. Unlike medicinal treatments, there is no consumption limit or adverse side effects. Therapy by Repeating Phrases of Positive Thoughts helps to confront the psywar trauma, phywar trauma and mental and psychological troubles. 
Keywords: Depression, Post-traumatic stress disorder, Psychological barrier, Brain, Neuroplasticity, Education-socialization, Syria, French.

\section{Introduction}

\section{Women and Education}

Women account the main society, because the positive education and socialization healthy start of their own. For this, this research is interested in the psychological health of women. In addition, all girls deserve equal access to health care, education and basic human needs. On this International Day of the Girl in October, this research has been done to demonstrate the support to girls and women around the world.

According to a study by Susan Nolen-Hoeksema, women are twice more numerous than men to ruminate when they are sad, anxious or depressed. According to her, this tendency is mainly explained by education. "From a young age, women are encouraged to express their emotions, to talk about their difficulties and to listen to others [1]. However, we make fun of a boy who is crying and you push him to react differently, less emotionally". This hyperemotivity push more willingly the feminine gender to keep examining and questioning their decisions. Women and men are not defined in the same way compared to the outside world.

\section{Mental Health Disorders, Depression and Women}

Mental health disorders were a complex syndrome characterized by pain, and by specific physical suffering or psychological discomfort caused by these disorders. These disorders were most prevalent in women. The negative impact of mental health disorders, e.g. depression, on quality of life is evident, a fact that causes the presence of high levels of stress and anxiety, especially in women with this disease (depression).

MPSYT (mental and psychological troubles) (e.g. depression) are a disease that most often affects women than men (twice more affected than men) [2]. Twice women are diagnosed with depression [2,3].

According repetitive results in psychiatric epidemiology studies, psychological disorders and psychic disease, e.g. depression, stress and anxiety, are nearly twice as common in women compared with men [4-9]. This was further explained by Noorbala et al. who reported that social difficulties, physiological factors, and stress caused by the environment are the important risk factors for mental disorders among women [10].

Research has shown that the frequency of depression in women may be related to biology, life cycle, hormonal and psychosocial factors [11].

Females were more likely to report stress due to higher self-expectations and a feeling of lack of competence; women also tend to over-report medical and psychological symptoms [12]. Expressing their feelings may be one of the ways females cope with stressful events [7].

Women with depression are more likely to have feelings of sadness, worthlessness and excessive guilt $[13,14]$. During his life, one in five people suffers from depression, $21 \%$ of women and $13 \%$ of men [15]. Major depression can occur in $10-35 \%$ of women. One in five women will experience a depressive episode in his life [16].

Sex factor is also a significant factor in depressive vulnerability. The prevalence between the sexes is highly uneven. Indeed, depression affects in France two times more women than men [17,18] and in Syria [19,20]. Depression is the third largest of female morbidity while it is seventh in the men's morbidity [21]. Moreover, it was observed a higher frequency of depressive relapses in women than in men [22].

In addition, anxiety disorders and/or depression are common during pregnancy. Such conditions should always be supported, because the consequences can be harmful to mother and child during pregnancy, childbirth or after birth. Antidepressant treatments are so commonly prescribed for pregnant women and growing in recent years. Indeed, these antidepressants pass into the bloodstream of the fetus is thus exposed to the drug throughout the treatment. Several studies have already been conducted on the effects of antidepressants on brain development of the fetus and the health problems in children. The occurrence of disorders of the autism spectrum in particular is subject to special attention. The child may then have withdrawal symptoms (symptomes de sevrage) which vary depending on the drug [23-25]. Recently, a Danish team examined the link between taking an antidepressant during pregnancy and the occurrence of epilepsy in children. A cohort of 734,237 children born in Denmark between 1997 and 2008 was studied. Of these, 12,438 are born to mothers who took antidepressants during pregnancy, or the month before. Antidepressant use during pregnancy was associated with a higher risk of epilepsy among children. Children exposed to antidepressants have a higher risk of epilepsy $(+27 \%)$ than non-exposed children. Taking an antidepressant only in the months before pregnancy but not during pregnancy also increased the risk of epilepsy in children. In utero exposure to antidepressants and seems associated with an increased risk of epilepsy in children [24].

Psychopathological processes, e.g. depression and anxiety, are related both to the characteristics of the stressor event and to the cognitive processing used by the individual to interpret them, as central elements of stress. Therefore, it is evident the role of cognitive mediation in triggering the stress and its effects, as well as in its confrontation [9]. 
The positive cognitions can improve the treatment of depression, anxiety and stress among adults, their psychological well-being and quality of life. The TRPPT are proposed as a fast treatment against MPSYT. This effective tool can be offered in therapy and has great ease of implementation. The results show improved stress control capabilities, improved mood and anxiety. So TRPPT of intervention has proved beneficial for adults as students. More subjects repeat phrases with a very positive sense, more depression, anxiety and stress decrease. Unlike medicinal treatments, there is no consumption limit or adverse side effects [26].

The objective of this study was to explore the short-term effects of the treatment program (TRPPT: Therapy by Repeating Phrases of Positive Thoughts).

\section{Methods}

\section{Hypotheses}

The main hypothesis was to obtain a significant improvement in symptoms of depression after therapy, and possibly other dimensions of psychological functioning as symptomatology's anxious and stressed in women.

\section{Sample}

The total sample consisted of 20 women from the University of Damascus, aged 19 to 27 years and 4 women with MS (Multiple Sclerosis) from Damascus Hospital and Ibn Alnafees Hospital, aged 20 to 29 years. The French sample consisted of 33 women from the University of Caen Normandy, aged 18 to 23 years. Participation is voluntary. It was composed of two groups: experimental group (17 Syrian women and 15 French women) and control group (10 Syrian women and 18 French women). The method of RPPT involved generating positive thoughts related or not related to MPSYT. Participants received training in the designated method and then practiced it for one week, before attending for reassessment.

\section{Intervention and Participants}

This RPPT procedure was performed on students and MS patients in order to reduce the severity of psychological and emotional disorders (depression, anxiety stress and their symptoms) and to see the effects of this approach on health in general.

This study is a randomized controlled trial. After randomization, participations are divided into two groups. Experimental group was compared to a control group. The treatment took place over 7 days, based on RPPT exercises. Participation was voluntary and responses were anonymous.

For MS patients, this study received approval from the neurology service, Damascus Hospital and Ibn Alnafees Hospital. The Ethics and Research Committee of the Damascus Hospitals Complex approved the study protocol, informed consents were obtained from the participants after the aims and objectives of the study had been explained.

\section{Program of Treatment (TRPPT)}

The treatment program includes two active techniques: 1 . Repeat only the positive words (words in self-repetition) after receipt of the SMS sent by a psychologist; 2 . Repeat during one week ( 7 days) the three phrases of positive thoughts from 3 to $5 \mathrm{~min}$ per day. TRPPT includes a training of repetitions phrases with very positive sense. Before the first SMS, the students and MS patients have received education in the RPPT for $15 \mathrm{~min}$, led by a psychologist, with an explanation on the progress of research and measurement tools [26].

The guidelines should be taught only once in the first training session for about 10 to $15 \mathrm{~min}$. Thus, the instruction given on the observance of the RPPT is to repeat the three sentences at least three times, for 3 to $5 \mathrm{~min}$ from the morning awakening and evening before sleeping. In addition, these Phrases of Positive Thoughts (PPT) can be repeated mentally throughout the day during performing daily actions (eating, walking, wait urban transport; ride a bike, before an interview, etc.) [26].

\section{Ethical Review and Compliance with Ethical Standards}

This study received approval from the neurology services, Damascus Hospital and Ibn Alnafees Hospital. The Ethics and Research Committee of the Damascus Hospitals Complex approved the studied protocol; informed consents were obtained from the participants after the aims and objectives of the study have been explained. Human ethics committee approval protocol number was 5174/خ-07/2015/10 .

\section{Informed Consent}

All the persons who take part in this study have given the informed consent.

\section{Assessment of Clinical Symptoms and Automatic Thoughts}

All participants completed an assessment and clinical evaluation that utilized the following structured interview and questionnaires.

All Syrian participants completed the Automatic thoughts questionnaire- Arabic (ATQ-18-Ar) [27,28]; the Beck Depression Inventory-II (BDI-II) [29]; the Depression Anxiety Stress Scale-21(DASS-21) [30,31]; the Rosenberg self-esteem scale (RSES) [32] and the Satisfaction with life scale (SWLS) [33].

For ATQ-18, the validity and reliability of the Syrian version of the ATQ-18-Ar was demonstrated. Cronbach's alpha was 0.87 for the NT (Negative thought) total score and 0.85 for the PT (Positive thought) total score. The split-half reliability was 0.86 for the NT total score, 0.90 for the PT total score, and 0.81 for the ATQ-18-Ar total score. The results suggested that the ATQ-18-Ar and ATQ$18-\operatorname{Fr}[26]$ possessed high internal consistency. 
All French participants completed the Automatic thoughts questionnaire- French (ATQ-18-Fr) [26]; the Beck Depression Inventory-II (BDI-II) [29]; the Depression Anxiety Stress Scale-21(DASS-21) [30].

\section{Statistical Analysis}

Statistical analysis was performed on 57 participants [29 (25 women students and 4 MS patients) for the experimental group following the treatment of the RPPT and 28 women students for the control group not following the treatment of the RPPT]. The objective of the statistical analysis is to describe and assess changes in MPSYT associated with repeating phrases positive thoughts (RPPT). To evaluate the effectiveness of this procedure, a comparison of scores before and after (T1 $v s$. T2) was performed for both depression scales (BDIII and DASS-21), using a test of mean comparison Wilcoxon, considering the scale of DASS-21 as the main variable.

The effect size, using the coefficient " $r$ " of Cohen and standardized response mean between $\mathrm{T} 1$ and $\mathrm{T} 2$ were calculated.

The threshold of significance tests is 0.05 (confidence level). All statistical analysis was performed using $\mathrm{R}$ (programming language).

\section{Results}

\section{Results Analysis}

For 57 women: 25 (10 Syrian and 15 French) for the experimental group following the treatment of the RPPT and 28 (10 Syrian and 18 French) for the control group not following the treatment of the RPPT were analyzed. 4
MS women were analyzed: 4 for the experimental group following the treatment of the RPPT.

\section{Descriptive Results}

Regarding the experimental group, in total, 4 MS women, aged 20 to 29 years $(m=24.0$ years $( \pm 3.91))$ were included. 25 women: 10 Syrian (mean age of 21.30 (1.33 \pm ) years (19 to 24$)$; and 15 French (mean age of $19.93( \pm 1.27)$ years (18 to 22 ) were enrolled in this study.

Regarding the control group, a total of 28 women were enrolled in this study: 10 Syrian with a mean age of 22.20 $( \pm 2.65)$ years (19 to 27$)$; 18 French with a mean age of 19.33 ( \pm 1.74$)$ years (18 to 23$)$.

\section{TRPPT and MPSYT}

The treatment of the RPPT has an effect on the experimental group compared to a control group. The results show that there is a difference between the experimental group who followed the TRPPT and the control group did not follow the TRPPT. The TRPPT, thus, significantly reduces depression, anxiety and stress and confirms our hypothesis tested (H1).

In the experimental group (Syrian and French women and MS women), there is a significant reduction in stress, depression and anxiety following the TRPPT protocol $(\mathrm{p}<0.05)$ (Tables 1 and 2; Figures 1 and 2).

In the experimental group (Syrian women and MS women), participants also improved self-esteem and satisfaction of life following the RPPT protocol $(\mathrm{p}<0.05)$ (Table 1 and Figure 1).

Table 1 showed the control group in Syrian sample had very substantial worsening of depression. When we saw the results, we were surprised; it is really surprising, but that is probably

Table 1. Effect of TRPPT on MPSYT among Syrian women

\begin{tabular}{|c|c|c|c|c|c|c|c|c|c|c|}
\hline & \multicolumn{8}{|c|}{ Experimental group, RPPT $(\mathrm{N}=14)$} & \multirow{2}{*}{\multicolumn{2}{|c|}{$\begin{array}{c}\text { Control group ( } \mathrm{N}=10) \\
10 \text { Women students }\end{array}$}} \\
\hline & \multicolumn{8}{|c|}{4 MS patients 10 Women students } & & \\
\hline & $\begin{array}{c}\text { Pre-RPPT } \\
\text { (T1) } \\
\text { MS }\end{array}$ & $\begin{array}{c}\text { Post-RPPT } \\
\text { (T2) } \\
\text { MS }\end{array}$ & $\begin{array}{c}\text { ES } \\
\text { T1-T2 }\end{array}$ & SRM & $\begin{array}{c}\text { Pre-RPPT } \\
\text { (T1) } \\
\text { Stu }\end{array}$ & $\begin{array}{c}\text { Post-RPPT } \\
\text { (T2) } \\
\text { Stu }\end{array}$ & $\begin{array}{c}\text { ES } \\
\text { T1-T2 }\end{array}$ & SRM & $\begin{array}{l}\text { Pre } \\
\text { (T1) }\end{array}$ & $\begin{array}{l}\text { Post } \\
\text { (T2) }\end{array}$ \\
\hline NAT & $27.25(8.53)$ & $11.25(5.18)^{*}$ & 1.876 & 1.618 & $18.40(4.94)$ & $9.40(1.64)^{* * *}$ & 1.822 & 1.982 & $21.10(3.31)$ & $19.50(3.10)^{£}$ \\
\hline PAT & $20.50(11.44)$ & $37.50(3.41)^{*}$ & 1.486 & 2.053 & $17.20(9.76)$ & $38.30(4.83)^{* * *}$ & 2.162 & 1.903 & $21.20(4.31)$ & $20.50(3.43)^{\mathrm{t}}$ \\
\hline Depression-BDI-II & $15.00(8.67)$ & $4.50(1.73)^{*}$ & 1.211 & 1.417 & $18.00(5.20)$ & $4.40(1.26)^{* * *}$ & 2.615 & 2.845 & $17.30(4.00)$ & $17.10(4.30)^{\mathrm{t}}$ \\
\hline Depression-DASS-21 & $9.75(7.84)$ & $2.75(1.70)^{*}$ & 0.893 & 1.098 & $14.00(4.39)$ & $3.80(1.03)^{* * *}$ & 1.962 & 2.138 & $13.30(3.36)$ & $12.80(2.65)^{t}$ \\
\hline Anxiety-DASS-21 & $12.50(7.04)$ & $2.00(1.63)^{*}$ & 1.492 & 1.848 & $14.80(4.46)$ & $4.60(1.07)^{* * *}$ & 2.286 & 2.487 & $13.40(2.83)$ & $14.00(3.39)^{\mathfrak{E}}$ \\
\hline Stress-DASS -21 & $13.25(7.27)$ & $4.50(3.31)^{*}$ & 1.169 & 1.330 & $14.80(4.02)$ & $5.30(2.35)^{* * *}$ & 2.363 & 2.393 & $14.20(2.97)$ & $14.60(2.17)^{£}$ \\
\hline RSES & $16.50(6.24)$ & $28.25(1.70)^{* *}$ & 1.883 & 2.095 & $16.60(5.07)$ & $26.80(2.04)^{* * *}$ & 1.676 & 2.546 & $19.40(4.29)$ & $15.8(4.31)^{\mathfrak{t}}$ \\
\hline SWLS & $16.50(7.04)$ & $25.50(0.57)^{*}$ & 1.278 & 1.282 & $17.20(5.39)$ & $25.10(1.19)^{* * *}$ & 1.781 & 1.775 & $20.60(2.95)$ & $18.10(3.14)^{£}$ \\
\hline
\end{tabular}

Means and standard deviations (in parentheses) obtained at baseline (T1) and after treatment (T2), with comparisons of means between $\mathrm{T} 1$ and $\mathrm{T} 2$ and calculation of effect sizes between T1 and T2

MPSYT: Mental and Psychological Troubles; TRPPT: Therapy by Repeating Phrases of Positive Thoughts; MS: Multiple Sclerosis; Stu: Students. NAT: Negative automatic thought; PAT: Positive Automatic Thought; DASS-21: Depression Anxiety Stress Scales-21; D: Depression; A: Anxiety; S: Stress; BDI-II: Beck Depression Inventory-II; RSES: Rosenberg Self-Esteem Scale; SWLS: Satisfaction with Life Scale

T1: First stage (initial assessment). T2: Second stage (revaluation). Pre- RPPT: Pre- Repeating Phrases of Positive Thoughts. PostRPPT: Post- Repeating Phrases of Positive Thoughts

${ }^{*} \mathrm{p}<0.05,{ }^{*} \mathrm{p}<0.01, * * * \mathrm{p}<0.001,{ }^{\mathrm{f}} \mathrm{p}>0.05$

p*: Wilcoxon Test; ES: Effect Size; SRM: Standardized Response Mean 
Table 2. Effect of TRPPT on MPSYT among French women

\begin{tabular}{|l|c|c|c|c|c|c|c|c|}
\hline & \multicolumn{4}{|c|}{ Experimental group, RPPT (N=15 Women students) } & \multicolumn{3}{c|}{ Control group (N=18 Women students) } \\
\hline & $\begin{array}{c}\text { Pre-RPPT } \\
\text { (T1) }\end{array}$ & $\begin{array}{c}\text { Post-RPPT } \\
\text { (T2) }\end{array}$ & $\mathbf{p}^{*}$ & ES T1-T2 & SRM & $\begin{array}{c}\text { Pre } \\
\text { (T1) }\end{array}$ & $\begin{array}{c}\text { Post } \\
\text { (T2) }\end{array}$ & $\mathbf{p}^{*}$ \\
\hline Depression-BDI-II & $10.73(8.77)$ & $9.60(8.85)$ & $\mathrm{p}<0,05$ & 0.106 & 0.325 & $8.83(7.17)$ & $7,33(4.60)$ & $\mathrm{p}>0,05$ \\
\hline Depression-DASS-21 & $5.53(5.66)$ & $3.73(4.33)$ & $\mathrm{p}<0,05$ & 0.326 & 0.478 & $2.83(2.83)$ & $2,11(3.04)$ & $\mathrm{p}>0,05$ \\
\hline Anxiety-DASS-21 & $5.93(5.57)$ & $3.53(4.10)$ & $\mathrm{p}<0,05$ & 0.405 & 0.816 & $4,11(3.17)$ & $3,55(3.79)$ & $\mathrm{p}>0,05$ \\
\hline Stress-DASS-21 & $8.60(5.08)$ & $6.20(5.19)$ & $\mathrm{p}<0,05$ & 0.279 & 0.599 & $5.38(3.36)$ & $5.16(3.83)$ & $\mathrm{p}>0,05$ \\
\hline
\end{tabular}

Means and standard deviations (in parenthesis) obtained at baseline (T1) and after treatment (T2), with comparisons of means between $\mathrm{T} 1$ and $\mathrm{T} 2$ and calculation of effect sizes between $\mathrm{T} 1$ and $\mathrm{T} 2$

MPSYT: Mental and Psychological Troubles; TRPPT: Therapy by Repeating Phrases of Positive Thoughts; T1: First Stage (initial assessment); T2: Second stage (revaluation); Pre-RPPT: Pre-Repeating Phrases of Positive Thoughts; Post-RPPT: Post- Repeating Phrases of Positive Thoughts

p*: Wilcoxon Test; ES: Effect Size; SRM: Standardized Response Mean

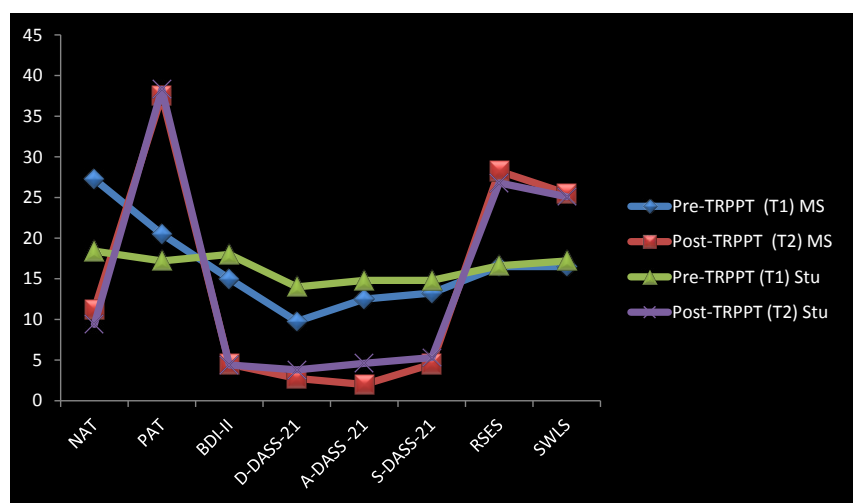

Figure 1. Diagram for effect of TRPPT on MPSYT among Syrian women

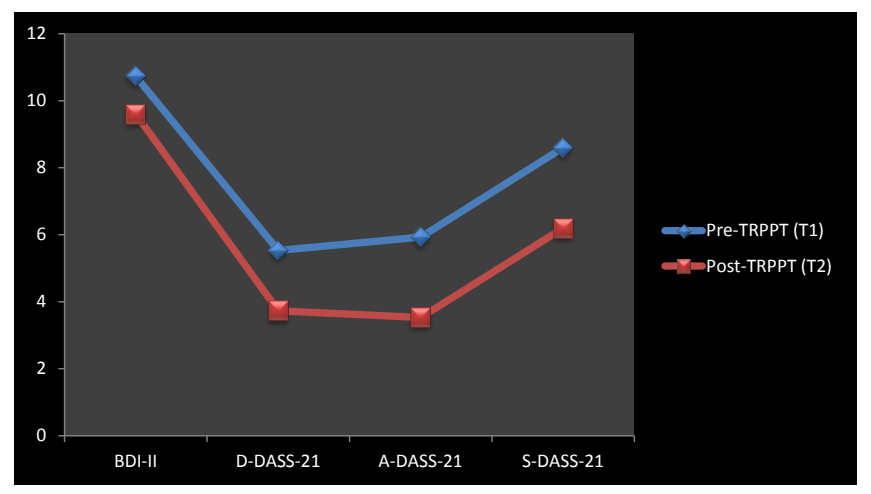

Figure 2. Diagram for effect of TRPPT on MPSYT among French women

reasonable because of the bad and abnormal conditions due to the war in Syria at the moment. The control group (10 Syrian women) subjected to abnormal conditions caused by the war causing the mental and psychological troubles. In additions, Table 2 showed the control group in French sample (18 French women) had of Anxiety.

\section{TRPPT and Confounding Factors}

This study assesses the effectiveness of psychotherapy (TRPPT), from population data in which TRPPT usage. Gender and/or age differences may influence the TRPPT of patients as well as their chances of recovery. In this scenario, gender and/or age confound the relation between TRPPT and recovery since gender and/or age is a cause of both TRPPT and recovery (Figure 3 ).

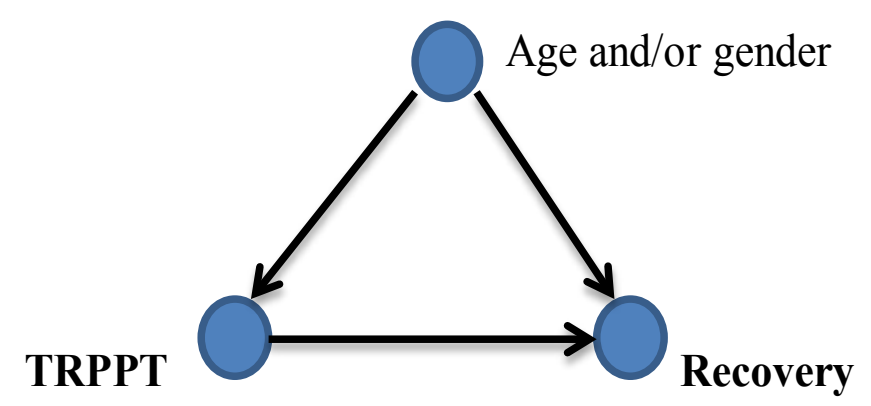

Figure 3. TRPPT and confounding factors

For that, a hierarchical multiple regressions and a multivariate regression analysis have performed in order to evaluate the role of confounding factors on final results, especially age and gender. For gender, in order to avoid if sex has an effect on the TRPPT, the study only takes homogeneous groups, only women. For age, the regression analysis indicates no significant effect concerning age of the participants. It appears, according to these results, that these two variables have no influence on the components of psychological disorders and well-being. According to regressions analysis, age has no influence $(p>0.05)$ on depression, anxiety, stress, self-esteem and satisfaction with life before or after the TRPPT. For future researches, it would be interesting to examine confounding factors, such as socio-economic factors for example.

\section{Discussion}

This research aimed to study the effects of the treatment of the RPPT on Syrian and French women subjected to MPSYT, very few people solicited so far in the literature.

The results of this research show effectiveness TRPPT protocol on the psychical and mental health of women (patients, students, adults).

Research has shown that the amendments on negative thoughts are ways to treat depression $[35,36]$. So, the results in this study showed that using RPPT are ways to treat depression and other MPSYT (ANX (Anxiety), STR (Stress), LSE (low self-esteem) and DSIL (dissatisfaction in life). According to these results, positive automatic thoughts and RPPT are absent in cognition depressive, anxious and stressed subjects. The presence of positive 
automatic thoughts and positive words is then an aspect of positivity in cognition that reduces MPSYT in anxious and depressive stressed subjects.

Before TRPPT, women (Syrian and French students and Syrian MS patients) have a high level of depression, anxiety, stress and a low level of self-esteem and satisfaction with life. After TRPPT, these women have a low level of depression, anxiety, stress and a high level of self-esteem and satisfaction with life. In other words, psychological and mental disorders decreased and psychological wellbeing increased with the TRPPT.

TRPPT introduce in women positive feelings such as comfortable, relax, optimism and the feelings of trust and hopefulness. These positive feelings support emotional vitality and happiness among Women who RPPT and reduce the common negative feelings and negative emotional states as depression, anxiety and stress.

TRPPT insures information of self-capacity and psychological barrier which led to positive cognitive appraisal in coping with stressful situations; and depressed, sad and worrying situations. TRPPT supports the positive self- image and positive future- image among women, which are the opposite images of those are common in depression.

\section{Conclusion}

This study suggests that RPPT treat the depression, stress and anxiety. The results show that TRPPT is important in treatment psychological disorders.

The TRPPT allows significantly reduce depressive symptoms, anxiety and stress, increases life satisfaction and self-esteem.

The TRPPT improves the quality of life, mental health and adaptation to the social and professional environment among women subjected to MPSYT, and MPSYT during war. The TRPPT used to help the women improve their concentration, confidence, self-esteem, regulate effort and academic and monitor cognitive and emotional reactions. TRPPT allows women to cope with psychological, emotional, environmental, social and romantic trauma, etc.

\section{Therapy by Repeating Phrases of Positive Thoughts (TRPPT): Comparison between Syrian and French samples}

Therapy by Repeating Phrases of Positive Thoughts proved its effectiveness under clinical and non-clinical conditions; and normal conditions such as stability in French and abnormal conditions as war in Syria. This experimental study has shown positive effects of TRPPT on the appraisal and treatment of MPSYT and stress, which determine our coping strategies.

In the world where negativity due to ignorance and lack of awareness and lack of reflection is prevailing everywhere in these moments because of the war, illiteracy, homeless, poverty, political conflicts and global geopolitical conflicts. Geopolitical situations in the world are alarming and people are under constant strains of bomb blasts. In these circumstances, there should be ways and measures to protect them from negativity.

In order to reduce the negativity, educationists and clinicians can initiate programs to cultivate positive thinking and positive words in their Women (e.g. students, patients, etc.). Positive thinking interventions should focus on how learners can identify their strengths, adopt new practices and habits and restructure their lives in ways that allow for a stream of positive emotions and experiences.

According to results in this study, TRPPT can be used by the counselors and clinicians to reduce the women's negativity by inducing positive thinking in them. They can change negative perceptions of the women into positive ones to make them healthy and productive citizens of stable and unstable countries because women are half of the world and play a vital role in this world.

\section{TRPPT and Positive Cognitive Triad}

The RPPT, which entail to do positive experiences against the MPSYT while childhood are "stored" in mnemonic structures that I call "positive neuro-cognitive schemes".

The scheme remains latent in human life. If, during his life, a person is faced with a similar situation to that which has been stored, the positive pattern will be reactivated. The scheme will then pass an unconscious state to a state similar to the consciousness that appears by positive automatic thoughts, positive words and RPPT.

Disappearance of depression associate with the existence of positive cognitions protogenic (example: "I know do this, so I trust me" instead of saying "I'm not doing that, so I'm terrible").

The RPPT is any mental activity that has a positive verbal content (ideas, judgments, self-instruction, and positive verbally articulated wishes).

The mechanism may be responsible for mental health and well-being is: The RPPT $\rightarrow$ Positive cognitive triad (automatic positive thinking) $\rightarrow$ self-positive schemes.

The positive cognitive triad responsible for mental health and symptoms of happiness is a positive view of self, environment and the future.

The RPPT $\rightarrow$ positive emotions $\rightarrow$ positive behavior $\rightarrow$ stable social relationship $\rightarrow$ families' stable $\rightarrow$ stable world.

When the positive words are activated, they trigger consistent emotions and behavioral and physiological responses harmonic and consistent.

The primary function of the RPPT is to promote energy conservation facing the perceived loss of resources. Over time, the RPPT reinforces positive thoughts, emotions and behavior that lead individuals to confront depression. 
These RPPT can help to stop the cycle of depression in women. The RPPT help interpret their environment and their interaction with others in a positive and realistic way.

The RPPT can help to change behaviors that may worsen depression. The RPPT, even at the most superficial level, play an essential role in the therapy of MPSYT because they directly influence the emotions and behavior of an individual.

\section{TRPPT and Depression and Its Symptoms}

The RPPT reduce emotional distress, loneliness, depressiveness, suicidal ideas that are higher in women. Women, following RPPT do not suffer from depression, are less likely to have feelings of sadness, worthlessness and excessive guilt, tired, irritable, lose interest in previously enjoyable activities and trouble sleeping.

\section{TRPPT and Resources}

Psychological, personal, family and environmental insufficient resources lead to a state of stress and/or anxiety and depression. On the other hand, the RPPT improve these psychological, personal, family and environmental resources and make sufficient to confront the state of stress and/or anxiety and depression. These RPPT also improve the personal resources include cognitive, emotional, behavioral and social among women.

The RPPT $\rightarrow$ positive emotions $\rightarrow$ positive behavior $\rightarrow$ love relationship $\rightarrow$ stable family.

\section{TRPPT and Stable Family}

In terms of family therapy and coping, women subjected to negative experiences (as war) and MPSYT with significant relational difficulties with family (as husband, children) are twice as likely as others to evoke a real psychological suffering and form a unstable family.

Unstable families have negative impacts on social and relational situation, especially in children. Therefore, these children will become husband and wife in the future. If these children were stable and not subjected to MPSYT and war, etc., these children will form the stable family and the world will become very secure. But if these children were unstable and subjected to MPSYT and war, etc., these children will form the unstable family and the world will become very dangerous.

These results show that TRPPT reduces the intensity of depression, also anxiety and stress and raises self-esteem, and satisfaction in life among women. This could well improve social and family relations between the family and also with their surroundings (e.g.: "There are many people who care about me").

\section{RPPT and Personality}

Thoughts and RPPT become the words $\rightarrow$ words eventually become the actions $\rightarrow$ acts eventually become the habits $\rightarrow$ habits eventually become the character and personality $\rightarrow$ character and personality eventually becomes the destiny $\rightarrow$ inevitably individual becomes what he thinks and what he repeat during the day and before the sleep.

\section{TRPPT and Age: Recommendations}

RPPT improves self-esteem and satisfaction with life. Thus, at the age of childhood, RPPT must begin to improve self-esteem and this is opposite those are common in depression. Then, at the age of adolescence, feelings of helplessness and hopelessness decrease in adolescents. Then, at the age of adults, agitation and other symptoms of depression do not appear.

So, RPPT must begin at an early age, i.e. from primary school or earlier. These RPPT help people (as family) to confront the bad conditions, psywar $^{1}$ trauma, phywar ${ }^{2}$ trauma and MPSYT, avoid unexpected situations like war, coup (e.g. military coup), accidents and deaths; and increase awareness and good citizenship culture among family and their children.

\section{TRPPT, Psychological Disorders, Neuroplasticity and Depression}

To diagnose and treat depression, we must study the cognitions [26,37]. In depression and Post-traumatic stress disorder (PTSD), neuroanatomical changes and cognitions were observed in the hippocampus, amygdala, and frontal cortex $[38,39]$. Depression can prevent neuroplasticity and lead to neurological damage [40]. In depressed or suffering from depression subjects, the results show a reduction in hippocampal volume by $19 \%$ and the left prefrontal cortex (PFC). Depressive subjects hyper-activation of the left amygdala and this activation is correlated with the severity of symptoms of depression.

\section{Positive Thoughts, Cognitive Impairment and Depression}

In the depression, impaired cognitive function was observed. This cognitive function is important in emotion regulation in depression, including negative automatic thoughts in depression or major depressive disorder (MDD). Research shows that positive automatic thoughts higher in subjects with depression would activate the prefrontal cortex (PFC), which is related to cognitive control [38].

\section{Positive and Negative Cognitions, Brain, Depression}

People with a high frequency of positive automatic thoughts showed a lower activation in the right superior temporal gyrus (STG) which is known to be associated with a negative emotional processing where NT is higher than the PT.

While those who have more positive thoughts than negative thoughts showed more activation in the left dorsolateral prefrontal cortex (DLPFC). In depression, the dorsolateral

${ }^{1}$ Psywar is the psychological consequence of torture and war violence as post-traumatic stress disorder (PTSD), fear, anxiety and major depressive episode (MDE).

${ }^{2}$ Phywar is the physical consequence of torture and war violence as distortions, deformation, loss of an arm or feet, etc. 
prefrontal cortex (DLPFC) is altered and the activation of the cortex decreases. Hypoactivity in the left dorsolateral prefrontal cortex is associated with rumination; people who have more automatic negative thoughts with positive automatic thoughts may be more likely to ruminate. Hypoactivity in the left dorsolateral prefrontal cortex (DLPFC) can help rumination facilitating inhibition of positive information and preventing the inhibition of negative information [38].

\section{Positives and Negatives Cognitions, Rumination, Depression}

Studies show that negative thoughts affect our professional, social, conjugal and personal lives. Mental rumination (overthinking) was identified as a new disorder of our time: "I should have ..."; "I could have ..."; "I'll never be able to ..." etc. these negative thoughts invade us, we run out and we make life impossible.

According to studies, $63 \%$ of young adults and $52 \%$ of forties (quadragénaires) have mental ruminations. This is a disturbing finding. But research has indicated that "we are all able to be positive, it would be only a matter of training" [41].

\section{Positives and Negatives Cognitions}

Negative thoughts (NT) are thoughts that imply criticism or self-devaluation. These thoughts dominate the cognition of a depressed person and make their very low psychological barrier. Their adaptation to everyday stressors becomes dysfunctional and they develop psychological and physical problems. Historically, psychologists have only focused on the negative mental states leading to the disease, disorder and unrest.

The influence of positive thinking on life satisfaction, quality of life and health outcomes has been generally neglected $[26,34,42,43]$.

\section{TRPPT, Context Processing and Processing Information}

The most common mental health problems among refugees are depression and post-traumatic stress disorder (PTSD) [44]. TRPPT is an effective treatment for depression, anxiety, stress, but also PTSD.

Mental health problems as depression, anxiety disorders, stress, low self-esteem, and dissatisfaction in life and posttraumatic stress disorder (PTSD) increase because of repeated and prolonged traumatization, worries about the future.

Individuals with depression, anxiety disorders, stress, low self-esteem and dissatisfaction in life and PTSD appear to suffer from a disruption of information processing on the context.

Processing information concerning the context implies the hippocampus (memory) and its relationship with the prefrontal cortex (treatment of high-level information) and amygdala (emotions).
For someone with a depression, stimuli associated with the trauma, such as an event or a failure as school failure triggers a fear response, anxiety attacks and a great stress even in very secure environments.

Studies have shown that the activity in these areas is disrupted in people with depression but also to PTSD $[38,39]$. The researchers estimate that a disruption in this circuit may interfere with the treatment of the context and explain many of the symptoms and much of the underlying biology.

A deficit in the context of the treatment would lead individuals with depression and/or anxiety disorders, PTSD to feel disconnected from the world around them and unable to shape their responses based on their current contexts. Their brains rather impose a context internalized in every situation, a context that is still waiting for the worst case, negativity and danger.

This type of deficit, occurring due to a combination of genetic, life experiences, psychological barrier and disrupted education and socialization wrong, can first create a vulnerability to indications of depression and posttraumatic stress. After trauma and/or depression, it would generate symptoms of hypervigilance, insomnia, negatives intrusive thoughts and dreams, repetitive negative thoughts and inappropriate emotional and physical reactions, violence against himself, women, children.

So, TRPPT may work by helping to anchor and adapt people in their current environment. TRPPT correct context processing and processing information in the brain.

TRPPT improve the processing information concerning the context implies the hippocampus and its relationship with the prefrontal cortex and amygdala.

\section{TRPPT and Psychological Barrier}

The psychological barrier is paramount and essential to confront the psychological problems and ensure good mental health and psychological well-being and general welfare. This barrier varies according to the particular personality of positive and negative person, education, and life experiences.

The psychological barrier is important to confront the problems and psychological trauma. Many people do not have this barrier or have a very low or low barrier.

The TRPPT have a major impact on the psychological barrier as well as psychological disorders and mental health.

The theory here is that the RPPT improve the psychological barrier of the individual.

The individual's education should be based on the RPPT and their positive impact on the brain and their improving mental, physical and general health... 


\section{RPPT, Talking with Self and Energy}

In other words, talking with self is a "positive assurances". When you think of something positive, you are attracting positive energy, and in turn to expel negative energy. The brain works in the way he is accustomed to think. In fact, the brain is a flexible member. The brain is affected by himself, so these similar thoughts, or repeated can come quickly, and this is called thinking style. Individual have to train your mind to think positively. Therefore, the individual also convert positive thinking into positive thoughts. For this, "We are reaping what we sowed"; even talk with yourself make it as a positive.

RPPT are a proven way to get rid of negative energy surrounding the individual that cause body fatigue, lethargy, laziness and all diseases.

\section{Clinical Implications and Research Implications}

The present study shows promising results for TRPPT; it's feasible, acceptable, and effective intervention in reducing PTSD and depression symptoms among Syrian and French samples in war and peace such as refugees in capital city...

This study is in accordance with the research of Alsaleh in 2016 [26] on Repeating Phrases of Positive Thoughts (RPPT) in France.

TRPPT has many important clinical implications:

- Deposits a valid and effective treatment among students and patients as MS.

- Proves its effectiveness under clinical and nonclinical conditions to treat of MPSYT and stress.

- Is an effective treatment in normal and abnormal conditions as war.

- Should be taught from childhood.

- The role of TRPPT in the initiation, maintenance, and treatment of depression in people is very important.

- Improves the brain activity, neuroplasticity and brain function as activity in the prefrontal and temporal cortices.

- Reinforces the love, stable families and social relationship.

- Enhances the self-esteem, satisfaction with life, and general well-being.

- Promotes relaxation and fun in inter- and intrapersonal.

- Reduces feelings of anger and social isolation.

- Improves social satisfaction and the frequency of pleasant events.

- Promotes quality of life because it has a significant impact on medical treatment and psychological treatment.
- Promotes also happiness and optimism.

- Improves academic success and reduce the fear of exams and the stress associated with this fear.

- Reduces trouble sleeping and improve the sleeping.

- Helps to come out of downward spiral of failure.

- TRPPT can and will be organized and focused on several areas: physiological (relaxation); cognitive (identification of cognitive schemas, negative thoughts, misinterpretation); emotional (decrease feelings of depression and anxiety and reduce the frequency of tantrums); and behavioral (improvement of social and communication skills).

- This study hypothesize that TRPPT can improve the brain functionally, context processing, processing information, neuroplasticity and reinforce psychological barrier and make it more solid. This study is in accordance with the research of Porto et al. on cognitive behavioral therapy (CBT) [38].

\section{Limitations}

In this study, there are a number of limitations. For Syrian samples, the main weakness is that it's not diagnosis of PTSD. For Syrian and French samples, the diagnosis and evaluation of MPSYT and their symptoms based on only self-report instruments.

Because of practical and logistical difficulties, this study have examined the short-term effects of TRPPT to reduce the PTSD, depression symptoms and other MPSYT compared to a control group.

For futures researches, it would be interesting to examine the medium and long-term effects of TRPPT, such as completed 4 weeks follow up (T3), 3 months follow up (T4), for example.

Besides the limitations of the current study, it is also important to note some highlights such as the samples studies, using the randomization to assign patients to groups. In addition, one of the positive points of this research is to have a control group but also clinical group. Additional, we evaluated PAT and NAT before applying the TRPPT and after applying the TRPPT for Syrian samples but not for French sample.

\section{Conflict of Interest}

The author declares that he has no competing interest.

\section{Acknowledgement}

The author thanks all the participants in this study.

\section{References}

1. Nolen-Hoeksema S, Harrell ZA. Rumination, depression, and alcohol use: Tests of gender differences. J Cogn Psycho 2002; 16: 391-403.

2. Chan Chee C, Beck F, Sapinho D, et al. La dépression en 
France - Enquête Anadep 2005, Saint-Denis : INPES, coll. Études santé 2009.

3. Lamoureux P, Briffault X, Caria A, et al. La dépression chez l'adulte, en savoir plus pour en sortir. Repérer les symptômes, connaître les traitements, savoir à qui s'adresser. INPES (Institut national de prévention et d'éducation pour la santé), Paris 2007

4. Cyranowski JM, Frank E, Young E, et al. Adolescent onset of the gender difference in life time rates of major depression. Archive of General Psychiatry 2000; 57: 21-27.

5. Piccinelli M, Wilkinson G. Gender differences in depression: Critical review. The British Journal of Psychiatry 2000; 117: 486-492.

6. Nolen-Hoeksema S. Gender differences in depression. Current Directions in Psychological Science 2001; 10: 173176.

7. Shamsuddin K, Fadzil F, Salwina W, et al. Correlates of depression, anxiety and stress among Malaysian university students. Asian Journal of Psychiatry 2013; 6: 318-323.

8. Bayram N, Bilgel N. The prevalence and socio-demographic correlations of depression, anxiety and stress among a group of university students. Social Psychiatry and Psychiatric Epidemiology 2008; 43: 667-672.

9. De Souza Ramiro F, Júnior IL, da Silva RCB, et al. Investigation of stress, anxiety and depression in women with fibromyalgia: A comparative study. Rev Bras Reumatol 2013; 54: 27-32.

10. Noorbala AA, Mohammad K, Bagheri Yazdi SA, et al. A view of mental health in Iran. Iranian Red Crescent Society Publications, Tehran 2001.

11. Eskandari F, Martinez PE, Torvik S, et al. Low bone mass in premenopausal women with depression. Archives of Internal Medicine 2007; 167: 2329-2336.

12. Vrana S, Lauterbach D. Prevalence of traumatic events and post-traumatic psychological symptoms in a nonclinical sample of college students. Journal of Traumatic Stress 1994; 7: 289-302.

13. Pollack WS. Mourning, melancholia and masculinity: Recognizing and treating depression in men. New Psychotherapy for Men 1998; 147-166.

14. Cochran SV, Rabinowitz FE. Men and depression: Clinical and empirical perspectives. Academic Press; San Diego 2000.

15. Nolen-Hoeksema S, Hilt LM. Gender differences in depression: III. Depression in specific populations ( $3^{\text {rd }}$ Edn) The Guilford Press: New York 2009.

16. Kessler RC, Ustun TB. The World Mental Health (WMH) survey initiative version of the World Health Organization (WHO) Composite International Diagnostic Interview (CIDI). Int J Methods Psychiatr Res 2004; 13: 93-121.

17. Sire D. Les troubles dépressifs: Données économiques et médico-économiques. L’Encéphale 2007; 33: 231-234.

18. Dassonville V. Du symptôme dépressif à la thérapeutique. L'Encéphale 2007; 33: 641-649.
19. Rudwan S. The Syrian list of symptoms: A field study. Journal of Social Science 2000; 28: 113-138.

20. Rudwan S. Beck depression inventory (BDI): Syrian version. King Saud University Journal 2003; 1: 453-486.

21. Gérard A, Raffaitin F, Cuche H. Dépression chez la femme. Les maladies dépressives: Medecine-Sciences Flammarion, Paris 1995; 84-90.

22. Reynaud M. Le modèle de l'attachement adulte dans la perturbation de la régulation émotionnelle et des liens affectifs des femmes hospitalisées souffrant de dépression. UFR Sciences Humaines, Laboratoire de Psychopathologie et de Psychologie Médicale, Université de Bourgogne 2011.

23. AFSSAPS (Agence Française de Sécurité Sanitaire des Produits de Santé). Bon usage des médicaments antidépresseurs dans le traitement des troubles dépressifs et des troubles anxieux de l'adulte 2006.

24. Mao Y, Pedersen LH, Christensen J, et al. Prenatal exposure to antidepressants and risk of epilepsy in childhood. Pharmacoepidemiol Drug Saf 2016.

25. Estelle B. Femme enceinte, prise d'antidépresseurs et épilepsie chez l'enfant. Santé sur le Net 2016.

26. Alsaleh M. Analyse psychosociale et cognitive de la santé mentale chez les étudiants de première année: - Validation du questionnaire des pensées positives et négatives et du questionnaire de la dépression de Beck: Effet des pensées positives et des facteurs psychosociaux. laboratoire du CERReV (EA 3918), Université de Caen Normandie 2016.

27. Hollon SD, Kendall PC. Cognitive self-statements in depression: development of an automatic thoughts questionnaire. Cognitive Therapy and Research 1980; 4: 383-395.

28. Kendall PC, Howard BL, Hays RC. Self-referent speech and psychopathology: The balance of positive and negative thinking. Cognitive Therapy and Research 1989; 13: 583598.

29. Beck AT, Steer RA, Brown GK. The psychological corporation, BDI-II Manual, London 1996.

30. Lovibond SH, Lovibond PF. Manual for the depression anxiety stress scales (2nd ed.) Psychology Foundation, Sydney 1995.

31. Taouk M, Lovibond PF, Laube R. Psychometric properties of an Arabic version of the depression anxiety stress scales (DASS21). Report for New South Wales Transcultural Mental Health Centre, Cumberland Hospital, Sydney 2001.

32. Rosenberg M. Society and the adolescent self-image. Wesleyan University Press, Middletown, CT 1989.

33. Diener E, Robert A, Emmons RJL, et al. The satisfaction with life scale. Journal of Personality Assessment 1985; 49: 71-75.

34. Alsaleh M, Lebreuilly R, Lebreuilly J, et al. The relationship between negative and positive cognition and psychopathological states in adults aged 18 to 20. Journal de Thérapie Comportementale et Cognitive 2016; 26: 79-90.

35. Beck AT, Rush AJ, Shaw BF, et al. Cognitive therapy of depression. Guilford Press, New York 1979. 
36. Hollon SD, Beck AT. Cognitive therapy of depression. Cognitive-behavioral intervention: Theory, research and procedures. Academic Press, New York 1979.

37. Feinstein A. Multiple sclerosis and depression. Multiple Sclerosis Journal 2011; 17: 1276-1281.

38. Koseki S, Noda T. The relationship between positive and negative automatic thought and activity in the prefrontal and temporal cortices: A multi-channel near-infrared spectroscopy (NIRS) study. Journal of Affective Disorders 2013; 151: 352-359.

39. Liberzon I, Abelson JL. Context processing and the neurobiology of post-traumatic stress disorder. Neuron 2016; 92.

40. Player MJ, Taylor JL, Weickert CS, et al. Neuroplasticity in depressed individuals compared with healthy controls. Neuropsychopharmacology 2013; 38: 2101-2108.
41. Desor D. Forums moi et ma santé à Nancy, "Halte aux pensées négatives". Conférences, Université de Lorraine, Nancy 2013.

42. Zarghuna N, et Ruhi K. Positive thinking in coping with stress and health outcomes: Literature review. Journal of Research and Reflections in Education 2010; 4: 42-61.

43. Alsaleh M, Lebreuilly R, Lebreuilly J, et al. Cognitive balance: States-of-mind model and mental health among French students. Best Practices in Mental Health 2015; 11: 42-53.

44. Acarturk C, Konuk E, Cetinkaya M, et al. EMDR for Syrian refugees with post-traumatic stress disorder symptoms: Results of a pilot randomized controlled trial. European Journal of Psychotraumatology 2015; 6: 27414.

\section{Correspondence to:}

Muaweah Ahmad Alsaleh,

PhD, Neuropsychologist, Psychotherapist,

Center for Research on Risks and Vulnerabilities, University of Caen Normandy \& CHU de Caen, France.

E-mail: moaouiya87@yahoo.com 